\title{
1 Supplement for
}

2 The formation of nitro-aromatic compounds under high $\mathrm{NO}_{\mathrm{x}^{-}}$ 3 anthropogenic VOCs dominated atmosphere in summer in Beijing, 4 China

5 Yujue Wang ${ }^{1}$, Min Hu ${ }^{*}{ }^{, 1,5}$, Yuchen Wang ${ }^{3}$, Jing Zheng ${ }^{1}$, Dongjie Shang ${ }^{1}$, Yudong Yang ${ }^{1}$, Ying Liu ${ }^{1,5}, \mathrm{Xiao} \mathrm{Li}^{1}$, 6 Rongzhi Tang ${ }^{1}$, Wenfei $\mathrm{Zhu}^{6}$, Zhuofei $\mathrm{Du}^{1}$, Yusheng $\mathrm{Wu}^{1}$, Song $\mathrm{Guo}^{1}$, Zhijun $\mathrm{Wu}^{1}$, Shengrong Lou ${ }^{6}$, Mattias 7 Hallquist ${ }^{2}$, and Jian Zhen $\mathrm{Yu}^{*}, 3,4$

$8 \quad{ }^{1}$ State Key Joint Laboratory of Environmental Simulation and Pollution Control, College of Environmental Sciences and 9 Engineering, Peking University, Beijing 100871, China

$10{ }^{2}$ Department of Chemistry and Molecular Biology, University of Gothenburg, Gothenburg, Sweden

$11{ }^{3}$ Environmental Science Programs, Hong Kong University of Science \& Technology, Hong Kong, China

$12 \quad{ }^{4}$ Department of Chemistry, Hong Kong University of Science \& Technology, Hong Kong, China

$13{ }^{5}$ Beijing Innovation Center for Engineering Sciences and Advanced Technology, Peking University, Beijing 100871, China

$14{ }^{6}$ Shanghai Academy of Environmental Sciences, Shanghai 200233, China

15 Correspondence to: Min Hu (minhu@pku.edu.cn); Jianzhen Yu (jian.yu@ust.hk) 


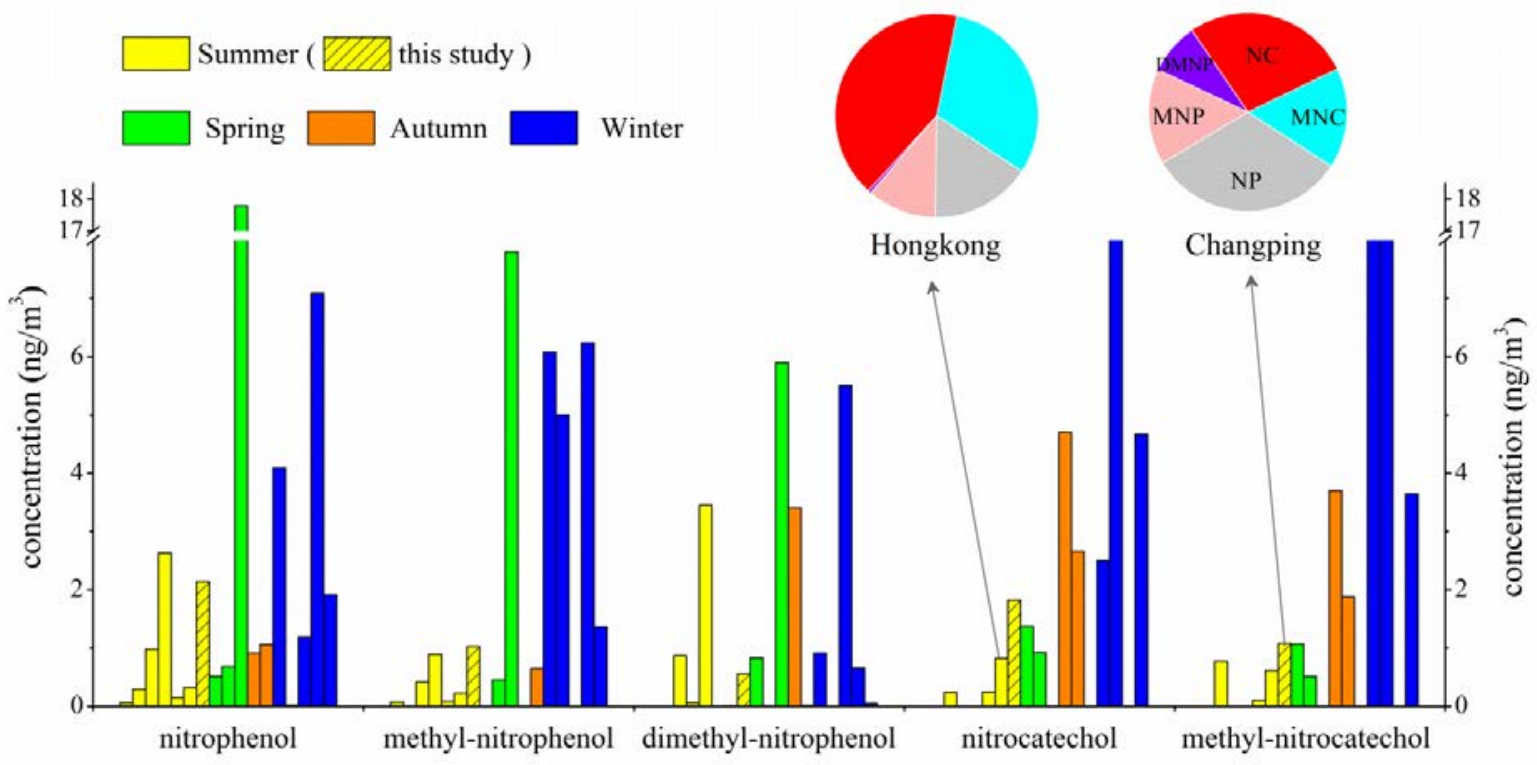

33 Figure S1 Comparison of individual NAC species across this and previous studies. For each species, the concentrations are 34 respectively reported in summer (yellow) in Melptitz, Germany (Teich et al., 2017), Flanders, Belgium (Kahnt et al., 2013), 35 Xianghe, China (Teich et al., 2017), Wangdu, China (Teich et al., 2017), Ljubljana, Slovenia (Kitanovski et al., 2012), Hong Kong, 36 China (Chow et al., 2016), Beijing, China (this study); in spring (green) in Flanders, Belgium (Kahnt et al., 2013), Hong Kong, 37 China (Chow et al., 2016), Rome, Italy (Cecinato et al., 2005); in autumn (orange) in Flanders, Belgium (Kahnt et al., 2013), 38 Hong Kong, China (Chow et al., 2016); and in winter (blue) in Melptitz, Germany (Teich et al., 2017), Detling, UK (Mohr et al., 39 2013), Flanders, Belgium (Kahnt et al., 2013), TROPOS, Germany (Teich et al., 2017) and Hong Kong, China (Chow et al., 2016) 40 from left side to right side. The inserted pie charts represent the NAC compositions in summer in Hong Kong (Chow et al., 2016) 41 and Changping, respectively. 

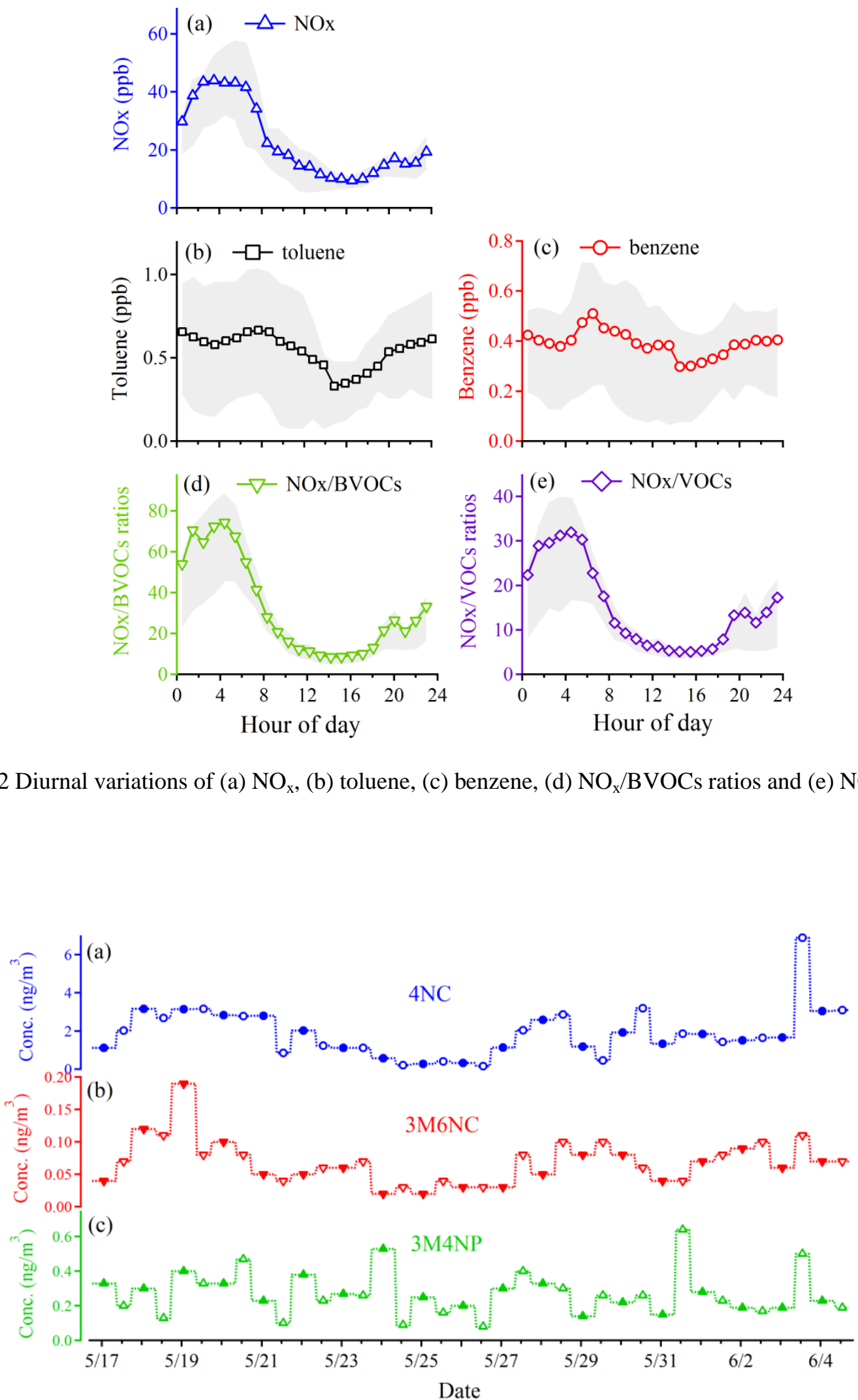

49 Figure S3 Time series of (a) 4-nitrocatechol (4NC), (b) 4-methyl-6-nitrocatechol (4M6NC), and (c) 3-methyl-450 nitrophenol (3M4NP). 


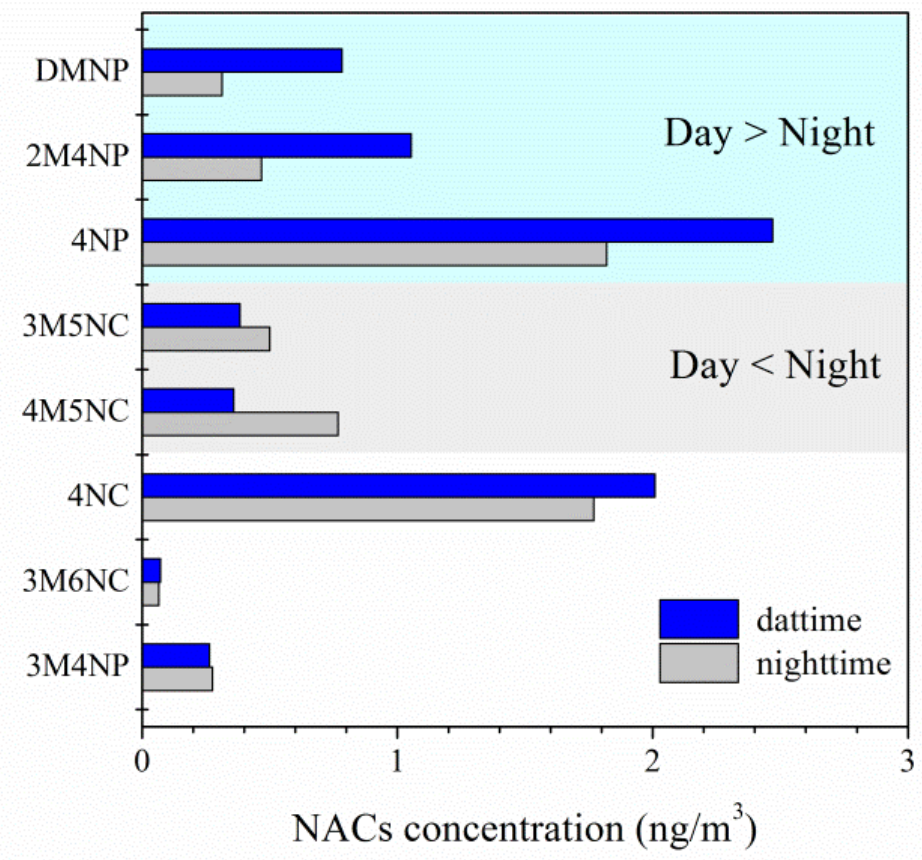

Figure S4 Day-night variations of NACs.

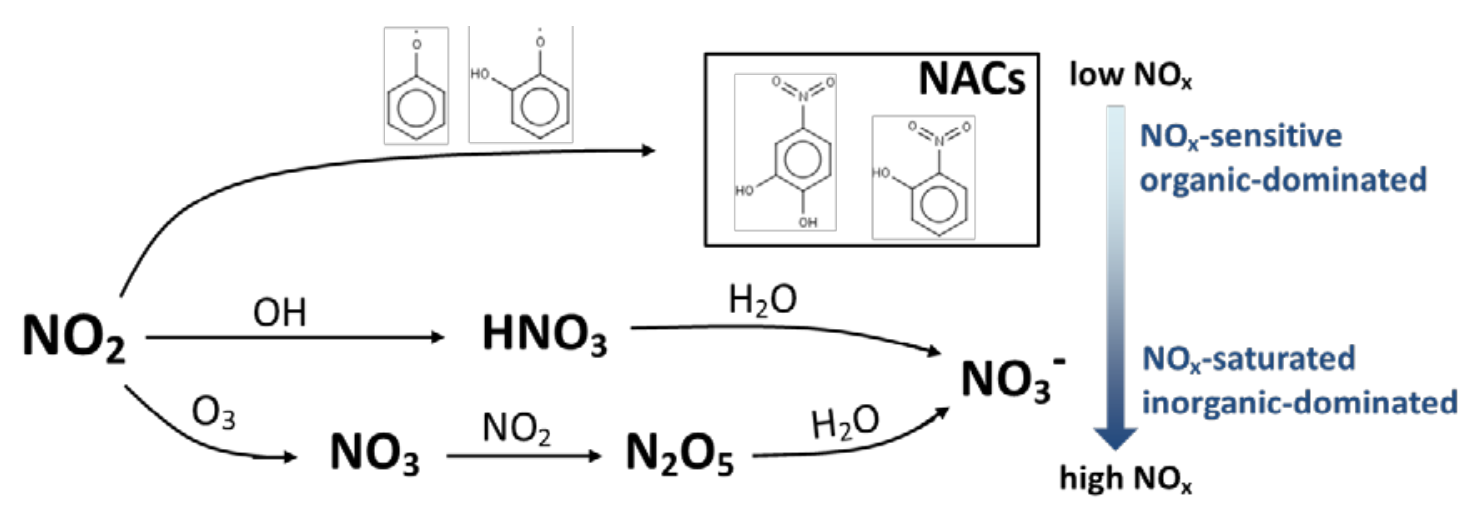

Figure S5 The schematic diagram and simplified mechanisms of the competing formation of inorganic nitrates and 
Table S1 Concentrations of nitroaromatic compounds in this and previous studies

\begin{tabular}{|c|c|c|c|c|c|c|c|c|c|c|c|}
\hline \multirow{2}{*}{ sites } & \multirow{2}{*}{ sampling period } & \multicolumn{9}{|c|}{ concentrations (ng/m³) } & \multirow{2}{*}{ references } \\
\hline & & 4NP (NP) & 3M4NP & 2M4NP & 2,6D4NP (DMNP) & 4NC (NC) & 4M5NC & 3M6NC & 3M5NC & Total & \\
\hline TROPOS, Germany & Jan-Feb, 2014 & 7.09 & 2.6 & 3.64 & 0.65 & & & & & 13.98 & (Teich et al., 2017) \\
\hline Melptitz, Germany & Jan-Feb, 2014 & 4.09 & 2.44 & 3.64 & 0.91 & & & & & 11.08 & (Teich et al., 2017) \\
\hline Maniz, Germany & May, 2006-June, 2007 & 2.48 & & & & 6.4 & & & & 8.88 & (Zhang et al., 2010) \\
\hline Melptitz, Germany & Jul, 2014 & 0.06 & 0.03 & 0.04 & n.d. & & & & & 0.13 & (Teich et al., 2017) \\
\hline Seiffen, Saxony, Germany & Oct, 2007-Mar, 2008 & & & & & & 2 & 2.9 & 0.37 & 5.27 & (Iinuma et al., 2010) \\
\hline Detling, UK & Jan-Feb, 2012 & 0.02 & \multicolumn{2}{|c|}{5} & & 2.5 & \multicolumn{3}{|c|}{8.2} & & (Mohr et al., 2013) \\
\hline Ljubljana, Slovenia & Dec, 2010-Jan, 2011 & 1.8 & 0.61 & 0.75 & & 75 & 29 & 6.2 & 34 & 147.36 & (Kitanovski et al., 2012) \\
\hline Ljubljana, Slovenia & Aug, 2010 & 0.15 & $<0.03$ & 0.05 & & 0.24 & 0.06 & $<0.03$ & 0.01 & 0.57 & (Kitanovski et al., 2012) \\
\hline Rome, Italy & Feb- Apr, 2003 & 17.8 & 7.8 & & 5.9 & & & & & 31.50 & (Cecinato et al., 2005) \\
\hline \multirow{4}{*}{$\begin{array}{c}\text { Hamme, Flanders, } \\
\text { Belgium }\end{array}$} & Spring , 2010 & 0.51 & & & 0.83 & 1.37 & \multicolumn{3}{|c|}{1.07} & 3.78 & \multirow{4}{*}{ (Kahnt et al., 2013) } \\
\hline & Summer, 2010 & 0.29 & & & 0.87 & 0.23 & \multicolumn{3}{|c|}{0.77} & 2.16 & \\
\hline & Autumn, 2010 & 0.91 & & & 3.4 & 4.7 & \multicolumn{3}{|c|}{3.7} & 12.71 & \\
\hline & Winter, 2010 & 1.19 & & & 5.5 & 11.6 & \multicolumn{3}{|c|}{13.9} & 32.19 & \\
\hline Los Angeles & May-June, 2010 & & \multicolumn{2}{|c|}{1.24} & 0.92 & 1.67 & \multicolumn{3}{|c|}{0.36} & 4.19 & (Zhang et al., 2013) \\
\hline \multirow{4}{*}{ Hong Kong } & Winter, 2009-2012 & 1.92 & 0.17 & 1.20 & 0.05 & 4.67 & 1.28 & 0.27 & 2.10 & 11.63 & \multirow{4}{*}{ (Chow et al., 2016) } \\
\hline & Spring, 2009-2012 & 0.68 & 0.06 & 0.39 & 0.01 & 0.92 & 0.17 & 0.10 & 0.24 & 2.57 & \\
\hline & Summer, 2009-2012 & 0.32 & 0.02 & 0.20 & 0.01 & 0.82 & 0.17 & 0.07 & 0.37 & 1.99 & \\
\hline & Antumn, 2009-2012 & 1.06 & 0.06 & 0.58 & 0.01 & 2.66 & 0.58 & 0.15 & 1.15 & 6.26 & \\
\hline Xianghe, China & Jul-Aug, 2013 & 0.98 & 0.09 & 0.32 & 0.06 & & & & & 1.45 & (Teich et al., 2017) \\
\hline \multirow{2}{*}{ Wangdu, China } & \multirow{2}{*}{ Jun, 2014} & 2.63 & 0.21 & 0.68 & 3.45 & & & & & 6.97 & (Teich et al., 2017) \\
\hline & & \multicolumn{8}{|c|}{$5.9^{\mathrm{a}}$} & 5.90 & (Wang et al., 2018) \\
\hline Jinan, China & Nov. 2013- Jan, 2014 & \multicolumn{8}{|c|}{$48.4^{\mathrm{a}}$} & 48.40 & (Wang et al., 2018) \\
\hline Jinan, China & Sep, 2014 & \multicolumn{8}{|c|}{$9.8^{\mathrm{a}}$} & 9.80 & (Wang et al., 2018) \\
\hline Yucheng, China & June, 2014 & \multicolumn{8}{|c|}{$5.7^{\mathrm{a}}$} & 5.70 & (Wang et al., 2018) \\
\hline Mt. Tai, China & July-Aug, 2014 & \multicolumn{8}{|c|}{$2.5^{\mathrm{a}}$} & 2.50 & (Wang et al., 2018) \\
\hline Beijing, China & May-Jun, 2016 & 2.15 & 0.27 & 0.76 & 0.55 & 1.89 & 0.56 & 0.07 & 0.44 & 6.62 & This study \\
\hline
\end{tabular}

Note: a: The identified NACs included 4-nitrophenol (4NP), 2-methyl-4-nitrophenol (2M4NP), 3-methyl-4-nitrophenol (3M4NP), 4-nitrocatechol (4NC),

62 4-methyl-5-nitrocatechol (4M5NC), 3-methyl-5-nitrocatechol (3M5NC), 3-methyl-6- nitrocatechol (3M6NC), 3-nitro-salicylic acid (3NSA), and5-nitro-salicylic acid (5NSA). 
63 Table S2 Pearson correlations between individual NAC species as well as their correlations with aerosol components,

64 aerosol surface area, VOC precursors, oxidants, $\mathrm{RH}$ and temperature $(\mathrm{n}=38)$

\begin{tabular}{|c|c|c|c|c|c|c|c|c|c|}
\hline & total NACs & $4 \mathrm{NP}$ & DMNP & 2M4NP & 3M4NP & $4 \mathrm{NC}$ & $3 \mathrm{M} 6 \mathrm{NC}$ & $3 \mathrm{M} 5 \mathrm{NC}$ & $4 \mathrm{M} 5 \mathrm{NC}$ \\
\hline total NACs & 1.00 & & & & & & & & \\
\hline $4 \mathrm{NP}$ & 0.88 & 1.00 & & & & & & & \\
\hline DMNP & 0.68 & 0.72 & 1.00 & & & & & & \\
\hline 2M4NP & 0.85 & 0.80 & 0.88 & 1.00 & & & & & \\
\hline 3M4NP & 0.63 & 0.56 & 0.70 & 0.59 & 1.00 & & & & \\
\hline $4 \mathrm{NC}$ & 0.92 & 0.69 & 0.42 & 0.71 & 0.41 & 1.00 & & & \\
\hline 3M6NC & 0.58 & 0.53 & 0.13 & 0.34 & 0.14 & 0.58 & 1.00 & & \\
\hline $3 \mathrm{M} 5 \mathrm{NC}$ & 0.76 & 0.54 & 0.23 & 0.37 & 0.45 & 0.80 & 0.59 & 1.00 & \\
\hline 4M5NC & 0.52 & 0.24 & $(0.08)$ & 0.05 & 0.34 & 0.60 & 0.49 & 0.84 & 1.00 \\
\hline $\mathrm{OM}$ & 0.62 & 0.62 & 0.11 & 0.38 & 0.17 & 0.65 & 0.49 & 0.57 & 0.46 \\
\hline $\mathrm{K}^{+}$ & 0.49 & 0.39 & 0.12 & 0.24 & 0.25 & 0.50 & 0.43 & 0.64 & 0.53 \\
\hline $\mathrm{SO}_{4}{ }^{2-}$ & 0.45 & 0.36 & 0.02 & 0.18 & 0.04 & 0.52 & 0.51 & 0.59 & 0.47 \\
\hline benzene & 0.64 & 0.57 & 0.23 & 0.39 & 0.23 & 0.68 & 0.34 & 0.67 & 0.48 \\
\hline toluene & 0.70 & 0.58 & 0.18 & 0.39 & 0.23 & 0.76 & 0.46 & 0.74 & 0.63 \\
\hline $\mathrm{NO}_{2}$ & 0.33 & 0.16 & $(0.17)$ & $(0.02)$ & 0.21 & 0.42 & 0.26 & 0.55 & 0.75 \\
\hline $\mathrm{O}_{3}$ & 0.34 & 0.43 & 0.37 & 0.49 & $(0.19)$ & 0.32 & 0.17 & 0.11 & $(0.31)$ \\
\hline $\begin{array}{c}\text { aerosol } \\
\text { surface area }\end{array}$ & 0.60 & 0.49 & 0.11 & 0.33 & 0.05 & 0.69 & 0.50 & 0.70 & 0.53 \\
\hline $\mathrm{RH}$ & 0.13 & $(0.06)$ & $(0.21)$ & (0.15) & 0.27 & 0.20 & 0.16 & 0.39 & 0.59 \\
\hline Temperature & 0.21 & 0.38 & 0.30 & 0.40 & $(0.32)$ & 0.17 & 0.16 & $(0.12)$ & $(0.45)$ \\
\hline
\end{tabular}

65

66

67

68

69

70

71

72 
74 Table S3 Pearson correlations between individual NAC species as well as their correlations with aerosol components, 75 aerosol surface area, VOC precursors, oxidants, RH and temperature during daytime and nighttime, respectively $76 \quad(n=19)$

\begin{tabular}{|c|c|c|c|c|c|c|c|c|c|}
\hline \multicolumn{10}{|l|}{ Daytime } \\
\hline & total NACs & $4 \mathrm{NP}$ & DMNP & 2M4NP & 3M4NP & $4 \mathrm{NC}$ & 3M6NC & $3 \mathrm{M} 5 \mathrm{NC}$ & 4M5NC \\
\hline total NACs & 1.00 & & & & & & & & \\
\hline $4 \mathrm{NP}$ & 0.90 & 1.00 & & & & & & & \\
\hline DMNP & 0.79 & 0.84 & 1.00 & & & & & & \\
\hline 2M4NP & 0.96 & 0.84 & 0.86 & 1.00 & & & & & \\
\hline 3M4NP & 0.80 & 0.87 & 0.94 & 0.82 & 1.00 & & & & \\
\hline $4 \mathrm{NC}$ & 0.92 & 0.68 & 0.51 & 0.84 & 0.54 & 1.00 & & & \\
\hline 3M6NC & 0.58 & 0.48 & 0.26 & 0.50 & 0.25 & 0.60 & 1.00 & & \\
\hline $3 \mathrm{M} 5 \mathrm{NC}$ & 0.83 & 0.72 & 0.49 & 0.67 & 0.54 & 0.85 & 0.63 & 1.00 & \\
\hline $4 \mathrm{M} 5 \mathrm{NC}$ & 0.85 & 0.64 & 0.41 & 0.75 & 0.49 & 0.92 & 0.68 & 0.85 & 1.00 \\
\hline $\mathrm{OM}$ & 0.69 & 0.63 & 0.32 & 0.57 & 0.35 & 0.69 & 0.55 & 0.73 & 0.80 \\
\hline $\mathrm{K}^{+}$ & 0.47 & 0.46 & 0.21 & 0.34 & 0.35 & 0.44 & 0.41 & 0.61 & 0.62 \\
\hline $\mathrm{SO}_{4}{ }^{2-}$ & 0.40 & 0.32 & 0.07 & 0.23 & 0.09 & 0.49 & 0.25 & 0.64 & 0.51 \\
\hline benzene & 0.75 & 0.68 & 0.50 & 0.62 & 0.51 & 0.72 & 0.46 & 0.89 & 0.75 \\
\hline toluene & 0.81 & 0.71 & 0.51 & 0.71 & 0.52 & 0.80 & 0.55 & 0.89 & 0.82 \\
\hline $\mathrm{NO}_{2}$ & 0.77 & 0.63 & 0.48 & 0.74 & 0.47 & 0.75 & 0.57 & 0.73 & 0.85 \\
\hline $\mathrm{O}_{3}$ & 0.38 & 0.35 & 0.03 & 0.21 & $(0.01)$ & 0.47 & 0.39 & 0.62 & 0.43 \\
\hline $\begin{array}{c}\text { aerosol } \\
\text { surface area }\end{array}$ & 0.57 & 0.46 & 0.19 & 0.42 & 0.20 & 0.65 & 0.50 & 0.79 & 0.73 \\
\hline $\mathrm{RH}$ & 0.30 & 0.15 & 0.10 & 0.24 & 0.11 & 0.36 & 0.21 & 0.47 & 0.55 \\
\hline $\mathrm{J}\left(\mathrm{O}^{1} \mathrm{D}\right)$ & $(0.72)$ & $(0.56)$ & (0.63) & $(0.76)$ & (0.67) & $(0.68)$ & $(0.14)$ & $(0.49)$ & $(0.60)$ \\
\hline Temperature & 0.10 & 0.17 & $(0.11)$ & 0.01 & $(0.16)$ & 0.17 & 0.25 & 0.10 & 0.01 \\
\hline \multicolumn{10}{|l|}{ Nighttime } \\
\hline & total NACs & $4 \mathrm{NP}$ & DMNP & 2M4NP & 3M4NP & $4 \mathrm{NC}$ & 3M6NC & 3M5NC & 4M5NC \\
\hline total NACs & 1.00 & & & & & & & & \\
\hline $4 \mathrm{NP}$ & 0.83 & 1.00 & & & & & & & \\
\hline DMNP & 0.23 & $(0.12)$ & 1.00 & & & & & & \\
\hline 2M4NP & 0.75 & 0.65 & 0.58 & 1.00 & & & & & \\
\hline 3M4NP & 0.27 & 0.01 & 0.60 & 0.46 & 1.00 & & & & \\
\hline $4 \mathrm{NC}$ & 0.97 & 0.79 & 0.12 & 0.65 & 0.11 & 1.00 & & & \\
\hline 3M6NC & 0.70 & 0.70 & $(0.15)$ & 0.40 & 0.07 & 0.68 & 1.00 & & \\
\hline
\end{tabular}




\begin{tabular}{|c|c|c|c|c|c|c|c|c|c|}
\hline 3M5NC & 0.91 & 0.59 & 0.24 & 0.53 & 0.34 & 0.88 & 0.63 & 1.00 & \\
\hline 4M5NC & 0.92 & 0.59 & 0.34 & 0.58 & 0.37 & 0.88 & 0.62 & 0.98 & 1.00 \\
\hline OM & 0.64 & 0.79 & $(0.31)$ & 0.50 & $(0.09)$ & 0.67 & 0.48 & 0.43 & 0.41 \\
\hline $\mathrm{K}^{+}$ & 0.66 & 0.46 & 0.12 & 0.57 & 0.18 & 0.67 & 0.44 & 0.68 & 0.61 \\
\hline $\mathrm{SO}_{4}^{2-}$ & 0.66 & 0.58 & 0.05 & 0.60 & $(0.02)$ & 0.68 & 0.65 & 0.56 & 0.53 \\
\hline benzene & 0.64 & 0.65 & $(0.04)$ & 0.57 & $(0.20)$ & 0.72 & 0.29 & 0.46 & 0.42 \\
\hline toluene & 0.79 & 0.76 & $(0.01)$ & 0.65 & $(0.16)$ & 0.86 & 0.45 & 0.60 & 0.58 \\
\hline $\mathrm{NO}_{2}$ & 0.60 & 0.56 & $(0.00)$ & 0.44 & $(0.05)$ & 0.61 & 0.37 & 0.52 & 0.55 \\
\hline $\mathrm{O}_{3}$ & 0.03 & 0.11 & $(0.19)$ & 0.11 & $(0.65)$ & 0.14 & $(0.04)$ & $(0.05)$ & $(0.11)$ \\
\hline $\begin{array}{c}\text { aerosol } \\
\text { surface area }\end{array}$ & 0.76 & 0.68 & 0.02 & 0.63 & $(0.17)$ & 0.82 & 0.52 & 0.62 & 0.59 \\
\hline $\mathrm{RH}$ & 0.46 & 0.37 & 0.59 & 0.77 & 0.62 & 0.33 & 0.34 & 0.31 & 0.34 \\
\hline Temperature & 0.02 & 0.23 & $(0.61)$ & $(0.17)$ & $(0.84)$ & 0.17 & 0.09 & $(0.06)$ & $(0.12)$ \\
\hline
\end{tabular}

77

78

79

80

81

82

83

84

85

86

87

88

89

90

91

92 


\section{References}

Cecinato, A., Di Palo, V., Pomata, D., Tomasi Sciano, M. C., and Possanzini, M.: Measurement of phase-distributed nitrophenols in Rome ambient air, Chemosphere, 59, 679-683, 10.1016/j.chemosphere.2004.10.045, 2005.

Chow, K. S., Huang, X. H. H., and Yu, J. Z.: Quantification of nitroaromatic compounds in atmospheric fine particulate matter in Hong Kong over 3 years: field measurement evidence for secondary formation derived from biomass burning emissions, Environ. Chem., 13, 665-673, 10.1071/en15174, 2016.

Iinuma, Y., Boge, O., Grafe, R., and Herrmann, H.: Methyl-nitrocatechols: atmospheric tracer compounds for biomass burning secondary organic aerosols, Environ. Sci. Technol., 44, 8453-8459, 10.1021/es102938a, 2010.

Kahnt, A., Behrouzi, S., Vermeylen, R., Safi Shalamzari, M., Vercauteren, J., Roekens, E., Claeys, M., and Maenhaut, W.: One-year study of nitro-organic compounds and their relation to wood burning in $\mathrm{PM}_{10}$ aerosol from a rural site in Belgium, Atmos. Environ., 81, 561-568, 10.1016/j.atmosenv.2013.09.041, 2013.

Kitanovski, Z., Grgic, I., Vermeylen, R., Claeys, M., and Maenhaut, W.: Liquid chromatography tandem mass spectrometry method for characterization of monoaromatic nitro-compounds in atmospheric particulate matter, J. Chromatogr., A, 1268, 35-43, 10.1016/j.chroma.2012.10.021, 2012.

Logan, J. A.: Nitrogen oxides in the troposphere - Global and regional budgets, J. Geophys. Res., [Oceans], 88, 10785-10807, 10.1029/JC088iC15p10785, 1983.

Mohr, C., Lopez-Hilfiker, F. D., Zotter, P., Prevot, A. S., Xu, L., Ng, N. L., Herndon, S. C., Williams, L. R., Franklin, J. P., Zahniser, M. S., Worsnop, D. R., Knighton, W. B., Aiken, A. C., Gorkowski, K. J., Dubey, M. K., Allan, J. D., and Thornton, J. A.: Contribution of nitrated phenols to wood burning brown carbon light absorption in Detling, United Kingdom during winter time, Environ. Sci. Technol., 47, 6316-6324, 10.1021/es400683v, 2013.

Teich, M., van Pinxteren, D., Wang, M., Kecorius, S., Wang, Z., Müller, T., Močnik, G., and Herrmann, H.: Contributions of nitrated aromatic compounds to the light absorption of water-soluble and particulate brown carbon in different atmospheric environments in Germany and China, Atmos. Chem. Phys., 17, 1653-1672, 10.5194/acp-17-1653-2017, 2017.

Wang, L., Wang, X., Gu, R., Wang, H., Yao, L., Wen, L., Zhu, F., Wang, W., Xue, L., Yang, L., Lu, K., Chen, J., Wang, T., Zhang, Y., and Wang, W.: Observations of fine particulate nitrated phenols in four sites in northern China: concentrations, source apportionment, and secondary formation, Atmos. Chem. Phys., 18, 4349-4359, 10.5194/acp-18-4349-2018, 2018. Zhang, X., Lin, Y. H., Surratt, J. D., and Weber, R. J.: Sources, composition and absorption Angstrom exponent of light-absorbing organic components in aerosol extracts from the Los Angeles Basin, Environ. Sci. Technol., 47, 3685-3693, 10.1021/es305047b, 2013.

Zhang, Y. Y., Müller, L., Winterhalter, R., Moortgat, G. K., Hoffmann, T., and Pöschl, U.: Seasonal cycle and temperature dependence of pinene oxidation products, dicarboxylic acids and nitrophenols in fine and coarse air particulate matter, Atmos. Chem. Phys., 10, 7859-7873, 10.5194/acp-10-7859-2010, 2010. 